\title{
The faithfulness of $\mathbf{F}_{\text {at }}$ : a proof-theoretic proof
}

\author{
Fernando Ferreira \\ Gilda Ferreira
}

\begin{abstract}
It is known that there is a sound and faithful translation of the full intuitionistic propositional calculus into the atomic polymorphic system $\mathbf{F}_{\text {at }}$, a predicative calculus with only two connectives: the conditional and the second-order universal quantifier. The faithfulness of the embedding was established quite recently via a model-theoretic argument based in Kripke structures. In this paper we present a purely proof-theoretic proof of faithfulness. As an application, we give a purely proof-theoretic proof of the disjunction property of the intuitionistic propositional logic in which commuting conversions are not needed.
\end{abstract}

\section{Introduction}

A propositional formula is a formula built from a stock of propositional letters (or constants) $P, Q, R$, etc using the propositional connectives $\perp, \wedge, \vee$ and $\rightarrow$. In [6], Prawitz defined the following translation:

$$
\begin{aligned}
& (P)^{\star}: \equiv P, \text { with } P \text { a propositional constant } \\
& (\perp)^{\star}: \equiv \forall X . X \\
& (A \rightarrow B)^{\star}: \equiv A^{\star} \rightarrow B^{\star} \\
& (A \wedge B)^{\star}: \equiv \forall X\left(\left(A^{\star} \rightarrow\left(B^{\star} \rightarrow X\right)\right) \rightarrow X\right) \\
& (A \vee B)^{\star}: \equiv \forall X\left(\left(A^{\star} \rightarrow X\right) \rightarrow\left(\left(B^{\star} \rightarrow X\right) \rightarrow X\right)\right),
\end{aligned}
$$

where $X$ is a second-order propositional variable which does not occur in $A^{\star}$ or $B^{\star}$. The target language is the language of Girard's (polymorphic) system $\mathbf{F}$ (cf. [5]). It consists of the smallest class of expressions which includes the atomic formulas (propositional constants $P, Q, R, \ldots$ and second-order propositional variables $X, Y, Z, \ldots)$ and is closed under implication and second-order universal quantification. Note that the translation $A^{\star}$ of a propositional formula $A$ is, clearly, a formula without second-order free variables. Prawitz's translation is actually an embedding of the propositional intuitionistic calculus into system $\mathbf{F}$ in the sense that if $\vdash_{i} A$ then $\vdash_{\mathbf{F}} A^{\star}$ (here $\vdash_{i}$ denotes provability in the intuitionistic propositional calculus and $\vdash_{\mathbf{F}}$ denotes provability in the system F). 
In 2006, the first author noticed (cf. [1]) that the above embedding still works if the target system $\mathbf{F}$ is restricted to a predicative system nowadays known as $\mathbf{F}_{\text {at }}$ (an acronym for atomic polymorphism). The atomic polymorphic system $\mathbf{F}_{\text {at }}$ has the same formulas as $\mathbf{F}$, but replaces the second-order universal elimination rule by a predicative variant. For definiteness, we describe the (natural deduction) rules of $\mathbf{F}_{\text {at }}$. The introduction rules are as in $\mathbf{F}$ :

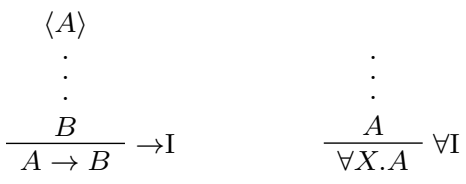

where the notation $\langle A\rangle$ says that the formula $A$ is being discharged and, in the universal rule, $X$ does not occur free in any undischarged hypothesis. The elimination rules of $\mathbf{F}_{\text {at }}$ are, however,

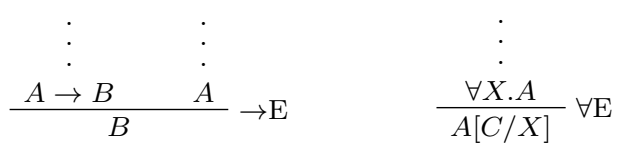

where $C$ is an atomic formula (free for $X$ in $A$ ), and $A[C / X]$ is the result of replacing in $A$ all the free occurrences of $X$ by $C$. Note that only atomic instantiations are permitted in the $\forall \mathrm{E}$ rule. This contrasts with the (impredicative) system $\mathbf{F}$, where $C$ can be any formula.

The reason why, despite the restriction of the $\forall$ E-rule, the system $\mathbf{F}_{\text {at }}$ is still able to embed full intuitionistic propositional calculus lies in the availability of instantiation overflow, i.e., for the three types of universal formulas occurring in Prawitz's translation, it is possible to derive in $\mathbf{F}_{\text {at }}$ the formulas resulting from instantiations of the second-order variable $X$ by any formula, not only the atomic ones. For a complete description of instantiation overflow and of the embedding see [1,2]. In the former reference, it is also shown that $\mathbf{F}_{\text {at }}$ has both the subformula property (for normal derivations) and an appropriate form of the disjunction property. (The notion of subformula only needs explanation for universal formulas. The proper subformulas of a formula of the form $\forall X . A[X]$ are the subformulas of the formulas of the form $A[C / X]$, for $C$ an atomic formula free for $X$ in $A$.) The latter reference is a study on the translation of the commuting conversions of the intuitionistic propositional calculus into $\mathbf{F}_{\text {at }}$. Note that, since the connectives $\perp, \vee$ and $\exists$ are absent from $\mathbf{F}_{\text {at }}$, this system has no commuting conversions. For more on $\mathbf{F}_{\text {at }}$, including a proof that the system is strongly normalizable for $\beta \eta$-conversions, see [3].

As we have discussed, Prawitz's translation $(\cdot)^{\star}$ gives a sound embedding of the intuitionistic propositional calculus into $\mathbf{F}_{\text {at }}$, that is: If $\vdash_{i} A$ then $\vdash_{\mathbf{F}_{\text {at }}} A^{\star}$. The translation is also faithful. I.e.:

$$
\text { If } \vdash_{\mathbf{F}_{\text {at }}} A^{\star} \text { then } \vdash_{i} A \text {. }
$$


This latter fact was recently proved using a model-theoretic argument in [4].

In the present paper, we give a pure proof-theoretic proof of the faithfulness of $\mathbf{F}_{\text {at }}$. We believe that this approach is interesting in its own right. Furthermore, it shows how to obtain a proof-theoretic proof of the disjunction property for the intuitionistic propositional calculus via natural deduction without the need of commuting conversions. As we have suggested in previous papers (cf. [2, 3]), the need for the ad hoc commuting conversions is a reflection of the fact that we are not considering intuitionistic propositional logic in its proper setting, viz the wider setting of $\mathbf{F}_{\text {at }}$.

The paper is organized in three sections. After this introduction, Section 2 presents the new proof-theoretic proof of the faithfulness of $\mathbf{F}_{\text {at }}$. The alternative proof of the disjunction property of the intuitionistic propositional calculus is presented in Section 3.

\section{A proof-theoretic proof of faithfulness}

A second-order universal formula which is a subformula of a formula of the form $A^{\star}$ ( $A$ a propositional formula) must take one of three forms: $\forall X . X$, $\forall X\left(\left(C^{\star} \rightarrow\left(D^{\star} \rightarrow X\right)\right) \rightarrow X\right)$ or $\forall X\left(\left(C^{\star} \rightarrow X\right) \rightarrow\left(\left(D^{\star} \rightarrow X\right) \rightarrow X\right)\right)$, with $C$ and $D$ propositional formulas. Hence, the following definition is in good standing:

Definition 2.1. Let $A$ be a propositional formula. For $B$ any subformula of $A^{\star}$, we define a formula $\tilde{B}$ in the language of propositional calculus $(\perp, \wedge, \vee, \rightarrow)$ extended with second-order variables (but without second-order quantifications) in the following way:

If $B$ is atomic, then $\tilde{B}: \equiv B$.

If $B: \equiv C \rightarrow D$, then $\tilde{B}: \equiv \tilde{C} \rightarrow \tilde{D}$.

If $B: \equiv \forall X$.X, then $\tilde{B}: \equiv \perp$.

If $B: \equiv \forall X\left(\left(C^{\star} \rightarrow\left(D^{\star} \rightarrow X\right)\right) \rightarrow X\right)$, then $\tilde{B}: \equiv C \wedge D$.

If $B: \equiv \forall X\left(\left(C^{\star} \rightarrow X\right) \rightarrow\left(\left(D^{\star} \rightarrow X\right) \rightarrow X\right)\right)$, then $\tilde{B}: \equiv C \vee D$.

Note that $B$ and $\tilde{B}$ have the same free variables. Also, when $C$ is a propositional formula, $\widetilde{C^{\star}}$ is just $C$.

Lemma 2.2. Let $\Gamma$ be a tuple of formulas in $\mathbf{F}_{\text {at }}$ and $A$ be a formula in $\mathbf{F}_{\text {at }}$ with their free variables among the variables in $\bar{X}$. If there is a proof (say $\mathcal{D}$ ) in $\mathbf{F}_{\text {at }}$ of $A[\bar{X}]$ from $\Gamma[\bar{X}]$ in which all formulas (occurring in $\mathcal{D}$ and $\Gamma[\bar{X}]$ ) are subformulas of formulas of the form $D^{\star}$ ( $D$ a propositional formula), then

$$
\tilde{\Gamma}[\bar{F} / \bar{X}] \vdash_{i} \tilde{A}[\bar{F} / \bar{X}]
$$


for any tuple of propositional formulas $\bar{F}$. For $\Gamma[\bar{X}]: \equiv A_{1}[\bar{X}], \ldots, A_{n}[\bar{X}]$, $\tilde{\Gamma}[\bar{F} / \bar{X}]$ denotes the tuple of propositional formulas $\tilde{A}_{1}[\bar{F} / \bar{X}], \ldots, \tilde{A}_{n}[\bar{F} / \bar{X}]$. (Of course, the reading of $\tilde{A}[\bar{F} / \bar{X}]$ is to first consider the transformed formula $\tilde{A}$ and, afterwards, effect the substitution $[\bar{F} / \bar{X}]$ in it. The alternative reading does not make sense in general.)

Proof. By induction on the length of the derivation $\mathcal{D}$.

If $\mathcal{D}$ is a one node proof-tree, then $A[\bar{X}]$ is in $\Gamma[\bar{X}]$. The result is trivial since for any tuple $\bar{F}$ of propositional formulas we have $\tilde{A}[\bar{F} / \bar{X}] \vdash_{i} \tilde{A}[\bar{F} / \bar{X}]$.

- Case where the last rule is a $\rightarrow \mathrm{I}$ :

$$
\begin{gathered}
\langle A[\bar{X}]\rangle \quad \Gamma[\bar{X}] \\
\vdots \\
\frac{B[\bar{X}]}{A[\bar{X}] \rightarrow B[\bar{X}]}
\end{gathered}
$$

Fix $\bar{F}$ a tuple of propositional formulas. The aim is to prove that $\tilde{\Gamma}[\bar{F} / \bar{X}] \vdash_{i}$ $\tilde{A}[\bar{F} / \bar{X}] \rightarrow \tilde{B}[\bar{F} / \bar{X}]$. According to the induction hypothesis, we have $\tilde{A}[\bar{F} / \bar{X}], \tilde{\Gamma}[\bar{F} / \bar{X}] \vdash_{i}$ $\tilde{B}[\bar{F} / \bar{X}]$. Thus, adding an introduction rule for implication which discharges $\tilde{A}[\bar{F} / \bar{X}]$, we get the desired result.

- Case where the last rule is a $\rightarrow \mathrm{E}$ :

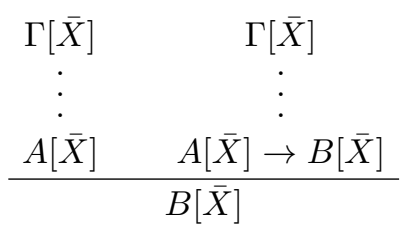

Fix $\bar{F}$ a tuple of propositional formulas. By induction hypothesis, we have both $\tilde{\Gamma}[\bar{F} / \bar{X}] \vdash_{i} \tilde{A}[\bar{F} / \bar{X}]$ and $\tilde{\Gamma}[\bar{F} / \bar{X}] \vdash_{i} \tilde{A}[\bar{F} / \bar{X}] \rightarrow \tilde{B}[\bar{F} / \bar{X}]$. Applying the elimination rule for implication, we get $\tilde{\Gamma}[\bar{F} / \bar{X}] \vdash_{i} \tilde{B}[\bar{F} / \bar{X}]$.

- Case where the last rule is a $\forall \mathrm{I}$ :

$$
\begin{gathered}
\Gamma[\bar{Y}] \\
\vdots \\
A[\bar{Y}, X] \\
\forall X . A[\bar{Y}, X]
\end{gathered}
$$

Since $\forall X . A[\bar{Y}, X]$ is a subformula of a translated formula $D^{\star}$, with $D$ a propositional formula, we know that only three cases may occur: (i) $A$ is $X$; (ii) $A$ has the form $\left(C^{\star} \rightarrow\left(E^{\star} \rightarrow X\right)\right) \rightarrow X$ or (iii) $A$ has the form $\left(C^{\star} \rightarrow\right.$ $X) \rightarrow\left(\left(E^{\star} \rightarrow X\right) \rightarrow X\right)$ with $C$ and $E$ propositional formulas. In any of the cases, the only free variable in $A$ is $X$. So, in the scheme above, $A[\bar{Y}, X]$ and $\forall X . A[\bar{Y}, X]$ may be replaced by $A[X]$ and $\forall X . A[X]$ respectively. 
In case (i), fix $\bar{F}$ a tuple of propositional formulas and let us prove that $\tilde{\Gamma}[\bar{F} / \bar{Y}] \vdash_{i} \perp$. By induction hypothesis we know that $\tilde{\Gamma}[\bar{F} / \bar{Y}] \vdash_{i} X[G / X]$ for every propositional formula $G$. Just take $G$ as being $\perp$.

In case (ii), we need to prove that $\tilde{\Gamma}[\bar{F} / \bar{Y}] \vdash_{i} C \wedge E$, for every tuple $\bar{F}$ of propositional formulas. Fix $\bar{F}$. By induction hypothesis, we know that $\tilde{\Gamma}[\bar{F} / \bar{Y}] \vdash_{i} \tilde{A}[G / X]$ for any propositional formula $G$. In particular, for $G: \equiv$ $C \wedge E$, we have

$$
\tilde{\Gamma}[\bar{F} / \bar{Y}] \vdash_{i}(C \rightarrow(E \rightarrow C \wedge E)) \rightarrow C \wedge E .
$$

Thus, in the natural deduction calculus for the intuitionistic propositional calculus, we have the following proof

$$
\begin{array}{cc}
\frac{\langle C\rangle\langle E\rangle}{C \wedge E} & \tilde{\Gamma}[\bar{F} / \bar{Y}] \\
\frac{E \rightarrow C \wedge E}{E \rightarrow(E \rightarrow C \wedge E)} & (C \rightarrow(E \rightarrow C \wedge E)) \rightarrow C \wedge E \\
\hline C \rightarrow(E \wedge E
\end{array}
$$

Therefore, $\tilde{\Gamma}[\bar{F} / \bar{Y}] \vdash_{i} C \wedge E$.

In case (iii), we need to prove that $\tilde{\Gamma}[\bar{F} / \bar{Y}] \vdash_{i} C \vee E$, for every tuple $\bar{F}$ of propositional formulas. Fix $\bar{F}$. By induction hypothesis, we know that $\tilde{\Gamma}[\bar{F} / \bar{Y}] \vdash_{i} \tilde{A}[G / X]$, for any propositional formula $G$. In particular, for $G: \equiv$ $C \vee E$, we have

$$
\tilde{\Gamma}[\bar{F} / \bar{Y}] \vdash_{i}(C \rightarrow C \vee E) \rightarrow((E \rightarrow C \vee E) \rightarrow C \vee E)
$$

Thus, in the intuitionistic propositional calculus, we have the following proof

$$
\begin{array}{ccc}
\frac{\langle C\rangle}{C \vee \bar{F} / \bar{Y}]} & \\
\frac{{ }_{C \rightarrow C \vee E}}{C \rightarrow C} & (C \rightarrow C \vee E) \rightarrow((E \rightarrow C \vee E) \rightarrow C \vee E) & \frac{\langle E\rangle}{C \vee E} \\
\hline & \frac{(E \rightarrow C \vee E) \rightarrow C \vee E}{E \rightarrow C \vee E} \\
\end{array}
$$

Therefore, $\tilde{\Gamma}[\bar{F} / \bar{Y}] \vdash_{i} C \vee E$.

- Case where the last rule is a $\forall \mathrm{E}$ :

$$
\begin{gathered}
\Gamma[\bar{Y}] \\
\vdots \\
\forall X . A[X, \bar{Y}] \\
A[C / X, \bar{Y}]
\end{gathered}
$$


with $C$ an atomic formula in $\mathbf{F}_{\text {at }}$, i.e., $C$ is a propositional constant or a secondorder variable. We assume w.l.o.g that if $C$ is a second-order variable then $C$ is among the variables $\bar{Y}$, say $Y_{i}$.

By hypothesis, since $\forall X . A[X, \bar{Y}]$ is a subformula of a translated formula, we know that this formula falls into one of the following three cases: (i) it is the translation of $\perp$; (ii) it is the translation of a conjunction; or (iii) it is the translation of a disjunction. Moreover, $\forall X . A[X, \bar{Y}]$ has no free variables and so, in the scheme above we can replace $\forall X . A[X, \bar{Y}]$ and $A[C / X, \bar{Y}]$ by $\forall X . A[X]$ and $A[C / X]$, respectively.

In case (i), we have the following proof in $\mathbf{F}_{\text {at }}$

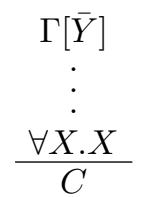

and we want to prove that $\tilde{\Gamma}[\bar{F} / \bar{Y}] \vdash_{i} C\left[F_{i} / Y_{i}\right]$, for any tuple $\bar{F}$ of propositional formulas. By $F_{i}$ we denote the formula of the tuple $\bar{F}$ which instantiates $Y_{i}$ in $\tilde{\Gamma}[\bar{F} / \bar{Y}]$.

Fix $\bar{F}$. By induction hypothesis we know that $\tilde{\Gamma}[\bar{F} / \bar{Y}] \vdash_{i} \perp$. As a consequence, in the intuitionistic propositional calculus we have the following proof

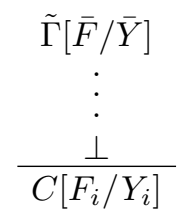

Hence, $\tilde{\Gamma}[\bar{F} / \bar{Y}] \vdash_{i} C\left[F_{i} / Y_{i}\right]$.

In case (ii), we have the following proof in $\mathbf{F}_{\text {at }}$

$$
\begin{gathered}
\Gamma[\bar{Y}] \\
\vdots \\
\frac{\forall X\left(\left(H^{\star} \rightarrow\left(E^{\star} \rightarrow X\right)\right) \rightarrow X\right)}{\left(H^{\star} \rightarrow\left(E^{\star} \rightarrow C\right)\right) \rightarrow C}
\end{gathered}
$$

We want to prove that $\tilde{\Gamma}[\bar{F} / \bar{Y}] \vdash_{i}\left(H \rightarrow\left(E \rightarrow C\left[F_{i} / Y_{i}\right]\right)\right) \rightarrow C\left[F_{i} / Y_{i}\right]$, for any tuple $\bar{F}$ of propositional formulas. Fix $\bar{F}$. By induction hypothesis we know that $\tilde{\Gamma}[\bar{F} / \bar{Y}] \vdash_{i} H \wedge E$. Thus, we have the following proof in the intuitionistic propositional calculus 


$$
\begin{array}{ccc} 
& \tilde{\Gamma}[\bar{F} / \bar{Y}] & \\
& \vdots & \tilde{\Gamma}[\bar{F} / \bar{Y}] \\
& \frac{H \wedge E}{H} & \vdots \\
\frac{E \rightarrow C\left[F_{i} / Y_{i}\right]}{C} & \frac{H \wedge E}{E} \\
\hline & C\left[F_{i} / Y_{i}\right] & \\
\left.\hline H \rightarrow\left(E \rightarrow C\left[F_{i} / Y_{i}\right]\right)\right) \rightarrow C\left[F_{i} / Y_{i}\right]
\end{array}
$$

This is what we want.

In case (iii), we have the following proof in $\mathbf{F}_{\text {at }}$

$$
\begin{gathered}
\Gamma[\bar{Y}] \\
\vdots \\
\frac{\forall X\left(\left(H^{\star} \rightarrow X\right) \rightarrow\left(\left(E^{\star} \rightarrow X\right) \rightarrow X\right)\right)}{\left(H^{\star} \rightarrow C\right) \rightarrow\left(\left(E^{\star} \rightarrow C\right) \rightarrow C\right)}
\end{gathered}
$$

Given any tuple $\bar{F}$ of propositional formulas, the aim is to show that $\tilde{\Gamma}[\bar{F} / \bar{Y}] \vdash_{i}$ $\left(H \rightarrow C\left[F_{i} / Y_{i}\right]\right) \rightarrow\left(\left(E \rightarrow C\left[F_{i} / Y_{i}\right]\right) \rightarrow C\left[F_{i} / Y_{i}\right]\right)$. Fix $\bar{F}$. By induction hypothesis, $\tilde{\Gamma}[\bar{F} / \bar{Y}] \vdash_{i} H \vee E$. Thus, we have the following proof in the intuitionistic propositional calculus

$$
\begin{array}{cccc}
\tilde{\Gamma}[\bar{F} / \bar{Y}] & \\
\vdots & \frac{\left\langle H \rightarrow C\left[F_{i} / Y_{i}\right]\right\rangle \quad\langle H\rangle}{C\left[F_{i} / Y_{i}\right]} \quad \frac{\left\langle E \rightarrow C\left[F_{i} / Y_{i}\right]\right\rangle}{C\left[F_{i} / Y_{i}\right]} & \langle E\rangle \\
\frac{C\left[F_{i} / Y_{i}\right]}{\left(E \rightarrow C\left[F_{i} / Y_{i}\right]\right) \rightarrow C\left[F_{i} / Y_{i}\right]} & \\
\frac{\left(H \rightarrow C\left[F_{i} / Y_{i}\right]\right) \rightarrow\left(\left(E \rightarrow C\left[F_{i} / Y_{i}\right]\right) \rightarrow C\left[F_{i} / Y_{i}\right]\right)}{(H)}
\end{array}
$$

We are done.

Theorem 2.3 (Faithfulness). Let $\Gamma: \equiv A_{1}, \ldots, A_{n}$ and $A$ be propositional formulas and consider their translations $\Gamma^{\star}: \equiv A_{1}^{\star}, \ldots, A_{n}^{\star}$ and $A^{\star}$ into $\mathbf{F}_{\text {at }}$.

If $\Gamma^{\star} \vdash_{\mathbf{F}_{\text {at }}} A^{\star}$ then $\Gamma \vdash_{i} A$.

Proof. Suppose that $\Gamma^{\star} \vdash_{\mathbf{F}_{\text {at }}} A^{\star}$. Since $\mathbf{F}_{\text {at }}$ has the normalization property (see $[3]$ ), we know that there is a proof, say $\mathcal{D}$, in normal form of $A^{\star}$ with premises $\Gamma^{\star}$. By the subformula property (see [1], page 5), all formulas that occur in $\mathcal{D}$ are subformulas of $A^{\star}$ or are subformulas of formulas in $\Gamma^{\star}$. Therefore, we are in the conditions of application of Lemma 2.2. Applying such lemma, we conclude that $\widetilde{\Gamma^{\star}} \vdash_{i} \widetilde{A^{\star}}$, i.e., $\Gamma \vdash_{i} A$. 


\section{Application}

An advantage of having a sound and faithful embedding between two systems is the possibility to transfer certain results from one system to the other. In this section, as an application of the (proof-theoretic proof of the) faithfulness of $\mathbf{F}_{\text {at }}$, we give a new proof of the disjunction property of the intuitionistic propositional calculus. Note that the usual proof-theoretic proof of the disjunction property requires the introduction of extra conversions associated with the connectives $\perp$ and $\vee$ : the so called commuting conversions or permutative conversions. They are needed to ensure that a proof in normal form has the subformula property. The proof-theoretic proof that we present below does not rely on commuting conversions.

Theorem 3.1. If $\vdash_{i} A \vee B$ then $\vdash_{i} A$ or $\vdash_{i} B$.

Proof. Suppose that $\vdash_{i} A \vee B$. Since the embedding of the full intuitionistic propositional calculus into $\mathbf{F}_{\text {at }}$ is sound, we have $\vdash_{\mathbf{F}_{\text {at }}}(A \vee B)^{\star}$. Applying the disjunction property of $\mathbf{F}_{\text {at }}$ (see [1], pages 5-7), we know that $\vdash_{\mathbf{F}_{\text {at }}} A^{\star}$ or $\vdash_{\mathbf{F}_{\text {at }}} B^{\star}$. By Theorem 2.3 (faithfulness), we conclude $\vdash_{i} A$ or $\vdash_{i} B$.

\section{References}

[1] Ferreira, F., 'Comments on predicative logic', Journal of Philosophical Logic, 35 (2006), 1-8.

[2] Ferreira, F., and G. Ferreira, 'Commuting conversions vs. the standard conversions of the "good" connectives', Studia Logica, 92 (2009), 6384 .

[3] Ferreira, F., and G. Ferreira, 'Atomic polymorphism', The Journal of Symbolic Logic, 78 (2013), 260-274.

[4] Ferreira, F., and G. Ferreira, 'The faithfulness of atomic polymorphism', in A. Indrzejczak, J. Kaczmarek, and M. Zawidzki, (eds.), Proceedings of Trends in Logic XIII, Łódź University Press, 2014, pp. 55-65.

[5] Girard, J.-Y., Y. Lafont, and P. Taylor, Proofs and Types, Cambridge University Press, 1989.

[6] Prawitz, D., Natural Deduction, Almkvist \& Wiksell, Stockholm, 1965. Reprinted, with a new preface, in Dover Publications, 2006. 